\title{
Construction of generalized rotations and quasi-orthogonal matrices
}

https://doi.org/10.1515/spma-2019-0010

Received April 2, 2019; accepted August 26, 2019

\begin{abstract}
We propose some methods for the construction of large quasi-orthogonal matrices and generalized rotations that may be used in applications in data communications and image processing. We use certain combinations of constructions by blocks similar to the one used by Sylvester to build Hadamard matrices. The orthogonal designs related with the matrix representations of the complex numbers, the quaternions, and the octonions are used in our construction procedures.
\end{abstract}

Keywords: Generalized rotations, quasi-orthogonal matrices, Hadamard matrices, orthogonal designs.

\section{Introduction}

Invertible matrices such that their inverse is easy to construct are very important in Linear Algebra and its many applications. Among such matrices the orthogonal matrices are certainly the most useful due to their nice algebraic and geometric properties. There are several generalizations of the orthogonal matrices described with names such as quasi-orthogonal matrices, almost orthogonal matrices, and generalized rotations. For example, matrices $A$ such that $A A^{\top}=w I$, where $I$ is the identity matrix and $w$ is a scalar, that usually depends on the size of $A$, are often called quasi-orthogonal matrices. A well-known class of such matrices is the set of Hadamard matrices. These are matrices whose entries are 1 or -1 , and that are the subject of extensive research. See [10] and the references therein.

In some applications it is important to use large matrices whose entries take only a small number of distinct values and the geometric properties that come from $A A^{\top}=w I$ may not be required. This happens in some applications in image and signal processing and data communication. See [1], [2], [11], and [12].

In this paper we present some simple procedures to construct large invertible matrices whose inverses are very easy to obtain without any arithmetical computations, with just transpositions of blocks and changes of signs. For example, block matrices of the form

$$
R=\left[\begin{array}{cc}
A & -B \\
B & A
\end{array}\right],
$$

that satisfy

$$
\left[\begin{array}{cc}
A & -B \\
B & A
\end{array}\right]\left[\begin{array}{cc}
A & B \\
-B & A
\end{array}\right]=\lambda I,
$$

where $\lambda$ is a nonzero scalar and $I$ denotes the identity matrix of the appropriate size. Note that the inverse of $R$ is a scalar multiple of the block-transpose of $R$. If the blocks $A$ and $B$ are symmetric then the block-transpose coincides with the transpose.

Some simple orthogonal designs appear in a natural way in our constructions. An orthogonal design of order $n$ and type $\left(c_{1}, c_{2}, \ldots, c_{k}\right)$ is a square matrix $C$ of order $n$ with entries in the set $\left\{0, \pm x_{1}, \pm x_{2}, \ldots, \pm x_{k}\right\}$,

^Corresponding Author: Luis Verde-Star: Department of Mathematics, Universidad Autónoma Metropolitana, Iztapalapa, Apartado 55-534, México D. F. 09340, México, E-mail: verde@xanum.uam.mx 
where the $x_{j}$ are commuting variables, that satisfies

$$
C C^{\top}=\left(\sum_{j=1}^{k} c_{j} x_{j}^{2}\right) I_{n}
$$

The study of orthogonal designs was initiated by Radon and Hurwitz around 1922 and is an active area of research. See [3], [4], [5], [6], [7], [8], and [9]. In this paper we use the orthogonal designs associated with the matrix representations of the complex numbers and the quaternions, and also an orthogonal design of order 8. We do not construct any new orthogonal designs.

The complex numbers can be represented by $2 \times 2$ matrices of real numbers. The starting idea behind our constructions is to replace the real numbers in the matrices that represent the complex numbers with some square matrices that satisfy certain conditions. This is done in Section 2 . We use a simple $2 \times 2$ block construction whose iteration allows us to obtain large matrices $A$ and $B$ that satisfy (1.1). In Section 3 we introduce some simple sequences of matrices of increasing size that can be used as building blocks for the large matrices. The entries of the building blocks take only a small number of different values. In Section 4 we use the matrix representations of the quaternions to obtain other block constructions, this time with $4 \times 4$ block matrices. We also use an orthogonal design to obtain an $8 \times 8$ block construction. Our methods may be used to obtain some sequences of Hadamard matrices of increasing size.

\section{Quasi-orthogonal matrices}

In this section we define some basic concepts, introduce the notation that will be used in this paper, and obtain some results about the construction of quasi-orthogonal matrices.

We denote by $\mathcal{M}_{n}$ the set of $n \times n$ matrices with complex entries and by $I_{n}$ the identity matrix in $\mathcal{M}_{n}$. The functions $c$ and $c^{*}$ are defined by the block constructions

$$
c(A, B)=\left[\begin{array}{cc}
A & -B \\
B & A
\end{array}\right], \quad c^{*}(A, B)=\left[\begin{array}{cc}
A & B \\
-B & A
\end{array}\right],
$$

where $A$ and $B$ are square matrices of the same size. They are functions from $\mathcal{M}_{n} \times \mathcal{M}_{n}$ to $\mathcal{M}_{2 n}$ for any positive integer $n$ and $c^{*}(A, B)=c(A,-B)$. Note that

$$
c(A, B) c(C, D)=c(A C-B D, A D+B C),
$$

and

$$
c(A, B) c^{*}(A, B)=c\left(A^{2}+B^{2}, B A-A B\right) .
$$

Definition. A matrix $R$ in $\mathcal{M}_{2 n}$ is quasi-orthogonal if and only if $R=c(A, B)$ for some matrices $A$ and $B$ in $\mathcal{M}_{n}$ and $c(A, B) c^{*}(A, B)=\lambda I_{2 n}$ for some nonzero number $\lambda$. A quasi-orthogonal matrix $R=c(A, B)$ for which $\lambda=1$ is called generalized rotation.

Using (2.2) we obtain immediately the following characterization of quasi-orthogonal matrices.

Lemma 2.1. A matrix $R=c(A, B)$ in $\mathcal{M}_{2 n}$ is quasi-orthogonal if and only if $A$ and $B$ commute and $A^{2}+B^{2}=\lambda I_{n}$ for some nonzero scalar $\lambda$.

If $c(A, B)$ and $c(C, D)$ are quasi-orthogonal and $A, B, C, D$ commute, then it is easy to see that $c(A, B)$ and $c(C, D)$ commute and the product $c(A, B) c(C, D)$ is quasi-orthogonal.

We define another pair of block constructions as follows. If $A$ and $B$ are square matrices of the same size define

$$
s(A, B)=\left[\begin{array}{ll}
A & B \\
B & A
\end{array}\right], \quad s^{*}(A, B)=\left[\begin{array}{cc}
A & -B \\
-B & A
\end{array}\right] .
$$


The maps $s$ and $s^{*}$ send each pair of matrices in $\mathcal{M}_{n} \times \mathcal{M}_{n}$ to a block-symmetric matrix in $\mathcal{M}_{2 n}$, for any positive integer $n$.

Multiplication of a matrix $T \in \mathcal{M}_{2 n}$ on the right by the matrix $s\left(0, I_{n}\right)$ permutes the columns of $n \times n$ blocks of $T$, and multiplication on the left permutes the rows of $n \times n$ blocks of $T$. Then we have

$$
s\left(0, I_{n}\right) c(A, B) s\left(0, I_{n}\right)=c^{*}(A, B), \quad A, B \in \mathcal{M}_{n},
$$

and therefore $c^{*}(A, B)$ is similar to $c(A, B)$, since $s\left(0, I_{n}\right)^{2}=I_{2 n}$. As a consequence, $c(A, B)$ and $c^{*}(A, B)$ have the same determinant and the same characteristic polynomial. Therefore, if $c(A, B) c^{*}(A, B)=\lambda I_{2 n}$ then $\operatorname{det}(c(A, B))^{2}=\lambda$.

Lemma 2.2. Let $A$ and $B$ be elements of $\mathcal{M}_{n}$ such that $A$ and $B$ commute and $A^{2}+B^{2}=\lambda I_{n}$ where $\lambda$ is a nonzero scalar. Then $s(A, B)$ and $s^{*}(A, B)$ commute and $s(A, B)^{2}+s^{*}(A, B)^{2}=2 \lambda I_{2 n}$.

Proof: Just note that $s(A, B)^{2}=s\left(A^{2}+B^{2}, 2 A B\right)$ and $s^{*}(A, B)^{2}=s\left(A^{2}+B^{2},-2 A B\right)$. From Lemmas 2.1 and 2.2 we obtain immediately the following result.

Theorem 2.1. Let $c(A, B) \in \mathcal{M}_{2 n}$ be quasi-orthogonal. Then $c\left(s(A, B), s^{*}(A, B)\right)$ is a quasi-orthogonal element of $\mathcal{M}_{4 n}$.

Let $A$ and $B$ be in $\mathcal{M}_{n}$. Using the maps $s$ and $s^{*}$ we define another construction by blocks as follows. Let $\sigma_{0}(A, B)=(A, B)$ and $\sigma_{1}(A, B)=\left(s(A, B), s^{*}(A, B)\right)$. For $m \geq 1$ define $\sigma_{m}(A, B)=\left(s\left(\sigma_{m-1}(A, B)\right), s^{*}\left(\sigma_{m-1}(A, B)\right)\right)$. Note that $\sigma_{m}(A, B) \in \mathcal{M}_{n 2^{m}} \times \mathcal{M}_{n 2^{m}}$. We write $\sigma_{m}(A, B)=$ $\left(\sigma_{m, 1}(A, B), \sigma_{m, 2}(A, B)\right)$. Let us note that $\sigma_{m, 1}(A, B)$ and $\sigma_{m, 2}(A, B)$ are block matrices and all of their blocks are in $\{A,-A, B,-B\}$.

Using Lemma 2.2 repeatedly and Lemma 2.1 we obtain the following theorem.

Theorem 2.2. Let $A$ and $B$ be elements of $\mathcal{M}_{n}$ such that $A$ and $B$ commute and $A^{2}+B^{2}=\lambda I_{n}$, where $\lambda \neq 0$. Then, for every nonnegative integer $m$ the matrices $\sigma_{m, 1}(A, B)$ and $\sigma_{m, 2}(A, B)$ commute and satisfy

$$
\left(\sigma_{m, 1}(A, B)\right)^{2}+\left(\sigma_{m, 2}(A, B)\right)^{2}=2^{m} \lambda I_{n 2^{m}},
$$

and therefore $c\left(\sigma_{m}(A, B)\right)$ is a quasi-orthogonal element of $\mathcal{M}_{n 2^{m+1}}$ constructed with blocks from the set $\{A,-A, B,-B\}$.

We say that $A$ and $B$ are the building blocks of the sequence of quasi-orthogonal matrices $c\left(\sigma_{m}(A, B)\right)$.

If $c(A, B)$ is quasi-orthogonal and $A$ and $B$ are symmetric then it is clear that $c^{*}(A, B)$ is equal to the transpose of $c(A, B)$ and therefore $c(A, B)$ is in fact a scalar multiple of an orthogonal matrix.

If $A$ and $B$ are symmetric elements of $\mathcal{M}_{n}$ then it is obvious that $s(A, B)$ and $s^{*}(A, B)$ are also symmetric. Therefore both components of $\sigma_{m}(A, B)$ are also symmetric for every $m \geq 0$, since $\sigma_{m}(A, B)$ is constructed by repeated application of the block constructions $s$ and $s^{*}$.

From the previous observations we obtain the following result.

Theorem 2.3. Let $A$ and $B$ be symmetric elements of $\mathcal{M}_{n}$ such that $A$ and $B$ commute and $A^{2}+B^{2}=\lambda I_{n}$, where $\lambda \neq 0$. Then, for every nonnegative integer $m$ the matrices $\sigma_{m, 1}(A, B)$ and $\sigma_{m, 2}(A, B)$ are symmetric and they commute and satisfy

$$
\left(\sigma_{m, 1}(A, B)\right)^{2}+\left(\sigma_{m, 2}(A, B)\right)^{2}=2^{m} \lambda I_{n 2^{m}},
$$

and therefore $c\left(\sigma_{m}(A, B)\right)$ is quasi-orthogonal and $(\sqrt{2})^{-m}(\sqrt{\lambda})^{-1} c\left(\sigma_{m}(A, B)\right)$ is an orthogonal generalized rotation and it is in $\mathcal{M}_{n 2^{m+1}}$. 


\section{Examples of sequences of building blocks}

If $A \in \mathcal{M}_{n}$ satisfies $A^{2}=\mu I_{n}$ we can take $B=A$ in Theorem 2.2 and then all the $n \times n$ blocks in the matrices $c\left(\sigma_{m}(A, A)\right)$ are either $A$ or $-A$.

For $n \geq 1$ let $U_{n} \in \mathcal{M}_{n}$ be the matrix that has all its entries equal to 1 . Now define

$$
V_{n}=\frac{2}{n} U_{n}-I_{n} .
$$

The entries in the diagonal of $V_{n}$ are all equal to $(2-n) / n$ and all the other entries are equal to $2 / n$. Therefore the sum of the entries in every row and every column is equal to 1.

Since $U_{n}$ is symmetric, so is $V_{n}$. A simple computation shows that $V_{n}^{2}=I_{n}$ and therefore $V_{n}$ is orthogonal and symmetric.

We also have

$$
\operatorname{det}\left(V_{n}\right)=(-1)^{n+1}, \quad \operatorname{det}\left(t I_{n}-V_{n}\right)=(t-1)(t+1)^{n-1},
$$

and

$$
\operatorname{det}\left((\sqrt{2})^{-m-1} c\left(\sigma_{m}\left(V_{n}, V_{n}\right)\right)\right)=1, \quad m \geq 0, n \geq 1 .
$$

Since $V_{n}$ is symmetric for every $n$, by Theorem 2.3, we see that the matrices $c\left(\sigma_{m}\left(V_{n}, V_{n}\right)\right)$ are scalar multiples of orthogonal matrices for every $n \geq 1$ and $m \geq 0$.

The matrix $V_{4}$ is interesting because

$$
2 V_{4}=\left[\begin{array}{cccc}
-1 & 1 & 1 & 1 \\
1 & -1 & 1 & 1 \\
1 & 1 & -1 & 1 \\
1 & 1 & 1 & -1
\end{array}\right],
$$

and therefore all the entries in the matrices $c\left(\sigma_{m}\left(2 V_{4}, 2 V_{4}\right)\right)$ are 1 or -1 and

$$
c\left(\sigma_{m}\left(2 V_{4}, 2 V_{4}\right)\right) c^{*}\left(\sigma_{m}\left(2 V_{4}, 2 V_{4}\right)\right)=4 * 2^{m+1} I_{4 * 2^{m+1}},
$$

and since $V_{4}$ is symmetric $c\left(\sigma_{m}\left(2 V_{4}, 2 V_{4}\right)\right)$ is a scalar multiple of an orthogonal matrix and therefore it is a Hadamard matrix. For example, $(1 / 8) c\left(\sigma_{3}\left(2 V_{4}, 2 V_{4}\right)\right)$ is an orthogonal element of $\mathcal{M}_{64}$, and $(\sqrt{2})^{-2 m+1} c\left(\sigma_{m}\left(2 V_{4}, 2 V_{4}\right)\right)$ is an orthogonal element of $\mathcal{M}_{2^{m+3}}$. Note that $2 V(4)$ is a circulant symmetric matrix.

If $n \neq 4$ and $n \neq 2$ then the entries of the matrices $c\left(\sigma_{m}\left(V_{n}, V_{n}\right)\right)$ take the four values $2 / n,-2 / n,(2-$ $n) / n,(n-2) / n$, for every $m \geq 0$. If $n=2$ the entries take the values $0,1,-1$.

We consider now another sequence of building blocks. Let

$$
Y_{n}=\frac{1}{n} U_{n}, \quad X_{n}=I_{n}-Y_{n}, \quad n \geq 1,
$$

where $U_{n}$ is as previously defined. The matrices $X_{n}$ and $Y_{n}$ are symmetric and singular and satisfy

$$
X_{n}^{2}=X_{n}, \quad Y_{n}^{2}=Y_{n}, \quad X_{n}+Y_{n}=I_{n}, \quad X_{n} Y_{n}=0, \quad n \geq 1 .
$$

Therefore $X_{n}$ and $Y_{n}$ commute and $X_{n}^{2}+Y_{n}^{2}=I_{n}$. By Theorem 2.3 the matrices $c\left(\sigma_{m}\left(X_{n}, Y_{n}\right)\right)$ are quasiorthogonal and are scalar multiples of orthogonal matrices for $n \geq 1$ and $m \geq 0$. Another property of these matrices is that

$$
\operatorname{det}\left((\sqrt{2})^{-m} c\left(\sigma_{m}\left(X_{n}, Y_{n}\right)\right)\right)=1, \quad m \geq 0, n \geq 1 .
$$

The entries of $c\left(\sigma_{m}\left(X_{n}, Y_{n}\right)\right)$ take four distinct values, except when $n=2$, in that case the values of the entries are $1 / 2$ and $-1 / 2$. For $m \geq 0$ the matrix $c\left(\sigma_{m}\left(2 X_{2}, 2 Y_{2}\right)\right)$ is a Hadamard matrix of size $2^{m+2}$. 


\section{Other block constructions}

The quaternions can be represented by $4 \times 4$ matrices of real numbers of the form

$$
q=\left[\begin{array}{cccc}
a & -b & -c & -d \\
b & a & d & -c \\
c & -d & a & b \\
d & c & -b & a
\end{array}\right] .
$$

The matrix that represents the conjugate quaternion $q^{*}$ is obtained by changing the sign of $b, c, d$. The product $q q^{*}$ is equal to $\left(a^{2}+b^{2}+c^{2}+d^{2}\right) I_{4}$ and represents the square of the norm of $q$.

We define the block constructions $t$ and $t^{*}$ as follows. If $A, B, C, D$ are in $\mathcal{M}_{n}$ define

$$
\begin{aligned}
t(A, B, C, D) & =\left[\begin{array}{cccc}
A & -B & -C & -D \\
B & A & D & -C \\
C & -D & A & B \\
D & C & -B & A
\end{array}\right], \\
t^{*}(A, B, C, D) & =\left[\begin{array}{cccc}
A & B & C & D \\
-B & A & -D & C \\
-C & D & A & -B \\
-D & -C & B & A
\end{array}\right] .
\end{aligned}
$$

Notice that if $A, B, C, D$ are symmetric then $t^{*}(A, B, C, D)$ is the transpose of $t(A, B, C, D)$.

From the properties of the matrix representation of the quaternions we obtain immediately the following result.

Lemma 4.1. If the matrices $A, B, C, D$ are in $\mathcal{M}_{n}$ and commute with each other then

$$
t(A, B, C, D) t^{*}(A, B, C, D)=\left(A^{2}+B^{2}+C^{2}+D^{2}\right) \otimes I_{4},
$$

where $\otimes$ denotes the Kronecker product.

From the previous Lemma we get the following theorem.

Theorem 4.1. If $A, B, C, D$ are in $\mathcal{M}_{n}$, commute with each other and satisfy $A^{2}+B^{2}+C^{2}+D^{2}=\lambda I_{n}$, with $\lambda \neq 0$, then $t(A, B, C, D)$ is an element of $\mathcal{M}_{4 n}$ and satisfies

$$
t(A, B, C, D) t^{*}(A, B, C, D)=\lambda I_{4 n} .
$$

It is easy to construct sets $\{A, B, C, D\}$ that satisfy $A^{2}+B^{2}+C^{2}+D^{2}=\lambda I_{n}$, with $\lambda \neq 0$, and such that no proper subset of $\{A, B, C, D\}$ satisfies the condition that the sum of the square of its elements is a nonzero multiple of the identity.

Le us note that equation (4.2) is similar to $c(A, B) c^{*}(A, B)=\lambda I_{2 n}$, which is the equation used to define quasi-orthogonal matrices. The matrix $t(A, B, C, D)$ can be written as

$$
t(A, B, C, D)=\left[\begin{array}{cc}
c(A, B) & -c^{*}(C, D) \\
c(C, D) & c^{*}(A, B)
\end{array}\right] .
$$

If we take $C=0$ and $D=0$ in (4.3) and substitute in (4.2) we see that (4.2) becomes the equation $c(A, B) c^{*}(A, B)=\lambda I_{2 n}$ written twice. Therefore if $c(A, B)$ is quasi-orthogonal then (4.2) is satisfied with $C=0$ and $D=0$. Therefore we can use (4.2) to extend the concept of quasi-orthogonal matrix. We use the same name and we say that $t(A, B, C, D)$ is quasi-orthogonal if it satisfies (4.2) with some $\lambda \neq 0$.

Using Theorem 4.1 and the properties of the block constructions $s$ ans $s^{*}$ it is easy to prove the following result. 
Theorem 4.2. Let $A, B, C$, D be elements of $\mathcal{M}_{n}$ that commute with each other and satisfy $A^{2}+B^{2}+C^{2}+D^{2}=\lambda I_{n}$, with $\lambda \neq 0$. Define

$$
\begin{array}{rlrl}
E_{1}:=s\left(s(A, B), s^{*}(C, D)\right), & & E_{2}:=s^{*}\left(s(A, B), s^{*}(C, D)\right), \\
E_{3}=s\left(s(C, D), s^{*}(A, B)\right), & E_{4}:=s^{*}\left(s(C, D), s^{*}(A, B)\right) .
\end{array}
$$

Then $E_{1}, E_{2}, E_{3}, E_{4}$ commute with each other and satisfy

$$
E_{1}^{2}+E_{2}^{2}+E_{3}^{2}+E_{4}^{2}=4 \lambda I_{4 n},
$$

and

$$
t\left(E_{1}, E_{2}, E_{3}, E_{4}\right) t^{*}\left(E_{1}, E_{2}, E_{3}, E_{4}\right)=4 \lambda I_{16 n} .
$$

The construction that produces the 4-tuple $\left(E_{1}, E_{2}, E_{3}, E_{4}\right)$ starting with $(A, B, C, D)$ can be iterated, in a way analogous to the definition of the map $\sigma_{m}$ in Section 2. We define $\gamma(A, B, C, D)=\left(E_{1}, E_{2}, E_{3}, E_{4}\right)$, with the $E_{j}$ defined in (4.4), and then define $\gamma_{0}(A, B, C, D)=(A, B, C, D), \gamma_{1}(A, B, C, D)=\gamma(A, B, C, D)$, and for $m \geq 1$ we define $\gamma_{m}(A, B, C, D)=\gamma\left(\gamma_{m-1}(A, B, C, D)\right)$. Note that $\gamma$ preserves the commutativity of its arguments and the property that the sum of the squares of its arguments is a nonzero multiple of the identity matrix.

Corollary 4.1. Let $A, B, C, D$ be as in the previous theorem and let $m \geq 0$. Then $t\left(\gamma_{m}(A, B, C, D)\right)$ is quasiorthogonal and

$$
t\left(\gamma_{m}(A, B, C, D)\right) t^{*}\left(\gamma_{m}(A, B, C, D)\right)=4^{m} \lambda I_{n * 4^{m+1}} .
$$

If $A, B, C, D$ are symmetric then $t\left(\gamma_{m}(A, B, C, D)\right)$ is a scalar multiple of an orthogonal matrix.

There are other block constructions that can be used instead of $t(A, B, C, D)$. For example,

$$
\left[\begin{array}{cccc}
A & -B & -C & -D \\
B & A & -D & C \\
C & D & A & -B \\
D & -C & B & A
\end{array}\right], \quad\left[\begin{array}{cccc}
A & B & C & D \\
B & -A & D & -C \\
C & -D & -A & B \\
D & C & -B & -A
\end{array}\right] .
$$

The first one corresponds to another matrix representation of the quaternions and is an orthogonal design.

Let $q(A, B, C, D)$ denote the first block matrix in (4.7) and let $q^{*}(A, B, C, D)=q(A,-B,-C,-D)$. We combine $t, t^{*}, q, q^{*}$ to obtain an $8 \times 8$ block matrix as follows. Define

$$
m(A, B, C, D, E, F, G, H)=\left[\begin{array}{ll}
q(A, B, C, D) & -t^{*}(E, F, G, H) \\
t(E, F, G, H) & q^{*}(A, B, C, D)
\end{array}\right],
$$

and let $m^{*}(A, B, C, D, E, F, G, H)=m(A,-B,-C,-D,-E,-F,-G,-H)$.

The proof of the following result is a straightforward computation.

Theorem 4.3. If the matrices $A, B, C, D, E, F, G, H \in \mathcal{M}_{n}$ commute with each other and satisfy $A^{2}+B^{2}+C^{2}+$ $D^{2}+E^{2}+F^{2}+G^{2}+H^{2}=\lambda I_{n}$, with $\lambda \neq 0$, then

$$
m(A, B, C, D, E, F, G, H) m^{*}(A, B, C, D, E, F, G, H)=\lambda I_{8 n} .
$$

Note that $m(A, B, C, D, E, F, G, H)+m^{*}(A, B, C, D, E, F, G, H)=2 A \otimes I_{8}$, where $\otimes$ denotes the Kronecker product.

Remark. The block construction $m(A, B, C, D, E, F, G, H)$ is related with an $8 \times 8$ orthogonal design, but it is not connected with the multiplication of the octonions, which is non-associative and therefore doesn't have a matrix representation.

If $A, B, C, D, E, F, G, H \in \mathcal{M}_{n}$ we define the 8 block matrices

$$
\begin{aligned}
& K_{1}=s\left(s\left(s(A, B), s^{*}(C, D)\right), s^{*}\left(s(E, F), s^{*}(G, H)\right)\right), \\
& K_{2}=s^{*}\left(s\left(s(A, B), s^{*}(C, D)\right), s^{*}\left(s(E, F), s^{*}(G, H)\right)\right),
\end{aligned}
$$




$$
\begin{gathered}
K_{3}=s\left(s^{*}\left(s(A, B), s^{*}(C, D)\right), s\left(s(E, F), s^{*}(G, H)\right)\right), \\
K_{4}=s^{*}\left(s^{*}\left(s(A, B), s^{*}(C, D)\right), s\left(s(E, F), s^{*}(G, H)\right)\right), \\
K_{5}=s\left(s\left(s^{*}(A, B), s(C, D)\right), s\left(s^{*}(E, F), s(G, H)\right)\right), \\
K_{6}=s^{*}\left(s\left(s^{*}(A, B), s(C, D)\right), s\left(s^{*}(E, F), s(G, H)\right)\right), \\
K_{7}=s\left(s^{*}\left(s^{*}(A, B), s(C, D)\right), s^{*}\left(s^{*}(E, F), s(G, H)\right)\right), \\
K_{8}=s^{*}\left(s^{*}\left(s^{*}(A, B), s(C, D)\right), s^{*}\left(s^{*}(E, F), s(G, H)\right)\right) .
\end{gathered}
$$

The map $\delta$ that sends $(A, B, C, D, E, F, G, H)$ to $\left(K_{1}, K_{2}, K_{3}, K_{4}, K_{5}, K_{6}, K_{7}, K_{8}\right)$ preserves commutativity and

$$
\sum_{j=1}^{8} K_{j}^{2}=8\left(A^{2}+B^{2}+C^{2}+D^{2}+E^{2}+F^{2}+G^{2}+H^{2}\right) .
$$

In the same way that we defined the iterates of $\sigma$ and $\gamma$ we can define the iterates of $\delta$, denoted by $\delta_{k}$, for $k \geq 0$.

Corollary 4.2. Let $A, B, C, D, E, F, G, H$ be as in the previous theorem and let $k \geq 0$. Then we have

$$
m\left(\delta_{k}(A, B, C, D, E, F, G, H)\right) m^{*}\left(\delta_{k}(A, B, C, D, E, F, G, H)\right)=8^{k} \lambda I_{n * 8^{k+1}} .
$$

It is clear that we can use the building blocks introduced in Section 3 to construct several kinds of large quasi-orthogonal matrices. In particular, we can construct some Hadamard matrices and some generalized Hadamard matrices involving complex numbers and quaternions.

Acknowledgement: The author thanks his colleagues from the Instituto de Matemáticas, Universidad Nacional Autónoma de México, for their hospitality during the time that this manuscript was written.

\section{References}

[1] Balonin, N.A., Sergeev, M.B., Quasi-Orthogonal local maximum determinant matrices, Appl. Math. Sci. (Ruse), 9 (2015) no. 6 , 285-293.

[2] Balonin, N., Sergeev, M., Quasi-orthogonal matrices with level based on ratio of Fibonacci numbers, Appl. Math. Sci. (Ruse), 9 (2015), no. 86, 4261-4268.

[3] Evangelaras, H., Georgiou, S. and Koukouvinos C., New orthogonal designs of order 56, J. Combin. Designs, 10 (2002) 387-393.

[4] Geramita, A.V., Geramita, J.M., Seberry Wallis, J., Orthogonal designs, Linear Multilinear Algebra, 3 (1976) 281-306.

[5] Ghaderpour, E., Constructions for orthogonal designs using signed group orthogonal designs, Discrete Math., 341(1) (2018) 277-285.

[6] Ghaderpour, E., Some constructions for amicable orthogonal designs, Australas. J. Combin., 63(3) (2015) 37-384.

[7] Goethals, J. M. and Seidel, J. J. , Orthogonal matrices with zero diagonal, Canad. J. Math, 19 (1967) 1001-1010.

[8] Kharaghani, H., Arrays for orthogonal designs, J. Combin. Des., 8(3) (2000) 166-173.

[9] Koukouvinos, C. and Seberry, J., Orthogonal Designs of Kharaghani Type: I, Ars Combin., 67, (2003) 89-96.

[10] Mitrouli, M., Sylvester Hadamard matrices revisited, Spec. Matrices, (2014) 2:120-124.

[11] Seberry, J., Spence, S.A., Wysocki, T.A., A construction technique for generalized complex orthogonal designs and applications to wireless communications, Linear Algebra Appl., 405 (2005) 163-176.

[12] Vostrikov, A., Sergeev, M., Balonin, N., and Chernyshev, S., Digital masking using Mersenne matrices and their special images, Procedia Comput. Sci., 112 (2017) 1151-1159. 